\title{
A Nest of LTR Retrotransposons Adjacent the Disease Resistance-Priming Gene NPR1 in Beta vulgaris L. U.S. Hybrid H20
}

\author{
David Kuykendall, Jonathan Shao, and Kenneth Trimmer \\ Molecular Plant Pathology Laboratory, ARS, USDA, Beltsville, MD 20705, USA \\ Correspondence should be addressed to David Kuykendall, david.kuykendall@ars.usda.gov \\ Received 26 September 2008; Accepted 25 January 2009 \\ Recommended by Cheng-Cang Wu
}

A nest of long terminal repeat (LTR) retrotransposons (RTRs), discovered by LTR_STRUC analysis, is near core genes encoding the NPR1 disease resistance-activating factor and a heat-shock-factor-(HSF-) like protein in sugarbeet hybrid US H20. SCHULTE, a 10833 bp LTR retrotransposon, with 1372 bp LTRs that are $0.7 \%$ divergent, has two ORFs with unexpected introns but encoding a reverse transcriptase with $r v e$ and Rvt2 domains similar to Ty1/copia-type retrotransposons and a hypothetical protein. SCHULTE produced significant nucleotide BLAST alignments with repeat DNA elements from all four families of plants represented in the TIGR plant repeat database (PRD); the best nucleotide sequence alignment was to ToRTL1 in Lycopersicon esculentum. A second sugarbeet LTR retrotransposon, SCHMIDT, 11565 bp in length, has 2561 bp LTRs that share 100\% identity with each other and share $98-99 \%$ nucleotide sequence identity over 10\% of their length with DRVs, a family of highly repetitive, relatively small DNA sequences that are widely dispersed over the sugarbeet genome. SCHMIDT encodes a complete gypsy-like polyprotein in a single ORF. Analysis using LTR_STRUC of an in silico deletion of both of the above two LTR retrotransposons found that SCHULTE and SCHMIDT had inserted within an older LTR retrotransposon, resulting in a nest that is only about $10 \mathrm{~Kb}$ upstream of NPR1 in sugarbeet hybrid US H20.

Copyright ( $) 2009$ David Kuykendall et al. This is an open access article distributed under the Creative Commons Attribution License, which permits unrestricted use, distribution, and reproduction in any medium, provided the original work is properly cited.

\section{Introduction}

Retrotransposons are now recognized as movers and shapers of plant genome evolution (see reviews $[1,2]$ ). That retrotransposon elements account for much of the sugarbeet (Beta vulgaris L.) genome was shown by the identification [3] of repetitive DNA sequences in Beta vulgaris similar to long interspersed nuclear elements (LINEs), a type of retrotransposon without long terminal repeats (LTRs), and other repetitive DNA sequences that resembled LTR retrotransposons of the Ty1-copia class. A repeated DNA sequence in Beta procumbens was described as "Athila-like" [4] since it was deduced to be part of a long terminal repeat with similarity to the Athila retrotransposon from Arabidopsis.

Prior to the present study, pDRV sequences [5] were known simply as a family of short highly amplified DNA repeats shown by fluorescent in situ hybridization (FISH) technique to be widely dispersed over all 18 chromosomes of sugarbeet.

Vulmarl, a mariner-class DNA transposon in Beta vulgaris [6], is $3909 \mathrm{bp}$, has $32 \mathrm{bp}$ terminal inverted repeats, and encodes, in a single ORF, a transposase with a characteristic "DDE" signature motif. Polymerase chain reaction (PCR) and fluorescent in situ hybridization (FISH) were used [6] to identify and to establish an abundance of En/Spm-like transposons in sugarbeet.

Coel, a DNA transposon within apparent LTRs and other retrotransposon-like features, was discovered on a sugarbeet genomic BAC carrying the NPR1 disease resistance-priming gene [7-9]. This recent discovery in Beta vulgaris of a unique $16.3 \mathrm{~Kb}$ CACTA En/Spm-like transposon named Coe1 [7] was followed by the finding of conserved microsynteny of NPR1 with another core plant gene whose predicted product has high similarity to a DNA-binding HSF protein [8]. About $70 \mathrm{~Kb}$ of repetitive DNA separates the HSF gene and 
NPR1 from another small core gene cluster with a CaMP gene specifying a signal peptide calmodulin-binding protein and a gene encoding a CK1-class protein kinase gene [8], greatly extending and disambiguating the results of the initial sequencing and partial in silico analysis of an NPR1 genecarrying sugarbeet BAC [9]. In summary, our laboratory has identified, sequenced, and annotated a bacterial artificial chromosome (BAC) carrying the NPR1 disease resistance priming gene of sugarbeet, Beta vulgaris L. [7-9].

Class I transposable elements which use reverse transcriptase to transpose via an RNA intermediate are termed retrotransposons. In order to identify possible LTR retrotransposons with LTRs, an intergenic region of repetitive DNA was examined by LTR_STRUC analysis, and this report details the discovery of a nest of retrotransposons about $10 \mathrm{~Kb}$ upstream from the NPR1 disease resistance gene in sugarbeet H20. This nest appears to have formed when both a copia-type and a gypsy-type elements inserted within an older LTR retrotransposon. Two full-length sugarbeet LTR retrotransposons are described herein for the first time.

\section{Materials and Methods}

Identification of a sugarbeet BAC carrying the NPR1 disease resistance control gene was described [9]. Genbank accession DQ851167 represents a partial sequence; the $38.6 \mathrm{~Kb}$ segment was the largest contig at that time. Subsequently the entire $130 \mathrm{~Kb}$ contiguous fragment was sequenced and annotated (Genbank accession EF101866). Basic methods used for DNA sequence analysis were described [9], and construction of the BAC library was detailed [10]. In the present study, LTR analyses of the NPR1 BAC were performed using LTR_STRUC [11], and LTR Finder [12]. Programs, einverted [13] (http://bioweb.pasteur.fr/seqanal/interfaces/einverted), and EMBOSS (http://emboss.sourceforge.net/) [13] were used to identify inverted repeats, and repeats were also found using NCBI BLAST (http://www.ncbi.nlm.nih.gov/BLAST/). An EST database for sugarbeet (http://genomics.msu.edu/ sugarbeet/blast.html) was employed for both nucleotide and protein BLAST to explore possible functional gene expression [14]. Subsequent analysis of DNA sequence data was performed using Lasergene version 6 (DNASTAR, Madison, Wis, USA). BLAST was used to identify the most similar protein products of LTR retrotransposons in other plant species. Multiple alignments were performed using MegAlign from the DNASTAR suite. Neighbor joining tree, or cluster analysis, was performed using MEGA 4 software (http://www.megasoftware.net/).

\section{Results and Discussion}

A genomic NPR1 disease resistance priming gene-carrying BAC [7-9] was subjected to LTR_STRUC and LTR FINDER analyses, and two distinct full-length LTR retrotransposons were identified (Figure 1). Depicted are RTR1 and RTR2, two LTR retrotransposons that we also term SCHULTE and SCHMIDT, respectively, as well as a previously described element, Coe1, a DNA transposase gene within apparent
LTRs and other retrotransposon-like features [7]. These repetitive DNA elements are intergenic, between two small clusters of core genes: HSF and NPR1 genes separated from $C a M P$ and $C K 1 P K$ genes encoding a signal peptide calmodulin-binding protein and a "casein kinase 1-class protein kinase," respectively.

SCHULTE, a 10833 bp long LTR retrotransposon, has $1372 \mathrm{bp}$ LTRs sharing only $99.3 \%$ nucleotide sequence identity. The $0.7 \%$ divergence in the LTRs of SCHULTE indicates about ten base substitutions occurred since insertion/transposition. This old, somewhat degraded retrotransposon has two ORFs encoding a Ty1/copia-like integrase/reverse transcriptase and a hypothetical protein (Figure 2). Unexpected introns, uncharacteristic of retrotransposon genes, may be the result of frameshifts and point mutations. SCHULTE has $98 \%$ nucleotide sequence identity over $\geq 9 \mathrm{~Kb}$ with a $9.7 \mathrm{~Kb}$ DNA fragment (DQ374026) and $1.3 \mathrm{~Kb}$ of a $5.3 \mathrm{~Kb}$ DNA fragment (DQ374025), each fragment of BAC62 [14]. BAC62 carries a Beta vulgaris L. genomic region adjacent a Beta procumbens translocation carrying a nematode resistance gene [15], thus BAC62 has a SCHULTE-like retrotransposon.

Named to honor an author of the first-described physical map of the afore-mentioned region, SCHULTE is the first full-length retrotransposon sequence from Beta vulgaris to be reported. Since two out of the three B. vulgaris BACs sequenced to date, BAC62 and the NPR1-carrying BAC, carry a SCHULTE-like element, there are likely a very large number of SCHULTE-like LTR retrotransposons in the sugarbeet genome. However, FLC, or the flowering control genecarrying BAC [16], did not carry a SCHULTE-like element.

SMART analysis showed that the predicted product of the SCHULTE reverse transcriptase gene has rve and $R v t 2$ protein domains. An alignment by MegAlign of the conserved rve and Rvt2 (Figure 3) domains of similar Ty1-copialike plant retrotransposon-encoded proteins, identified by BLAST, were analyzed by neighbor joining in MEGA 4 to assess structural relatedness (Figure 4). As shown in Figure 3, the predicted product of the Beta vulgaris SCHULTE reverse transcriptase gene has conserved rve and Rvt2 domains shared among highly similar domains of products of LTR retrotransposons from Medicago truncatula, Vitis vinifera, Oryza sativa japonica, Zea mays, and Glycine max. Except for the Solanum demissum and Vitis vinifera accessions, the $R v t 2$ domain evidently has a conserved YVDDIIF active site (Figure 3).

Similar LTR retrotransposon gene products in Arabidopsis thaliana, Solanum demissum, and particularly in Phaseolus vulgaris are structurally divergent (Figures 3 and 4). A search of the TIGR plant repeat database revealed that SCHULTE produced nucleotide sequence matches with many different copia-like retrotransposons in all four families: Brassicaceae, Fabaceae, Gramineae, and Solanaceae. The best PRD nucleotide sequence alignment match $(E=$ $3.8 \mathrm{e}^{-232}$ ) was to ToRTL1 in Lycopersicon esculentum.

Probable expression of integrase/reverse transcriptase gene(s) in active SCHULTE-like retrotransposon was shown by BLAST alignment $(E=0.0)$ of the ORF with BI643218, an EST, or expressed sequence tag. Expression of both LTRs 


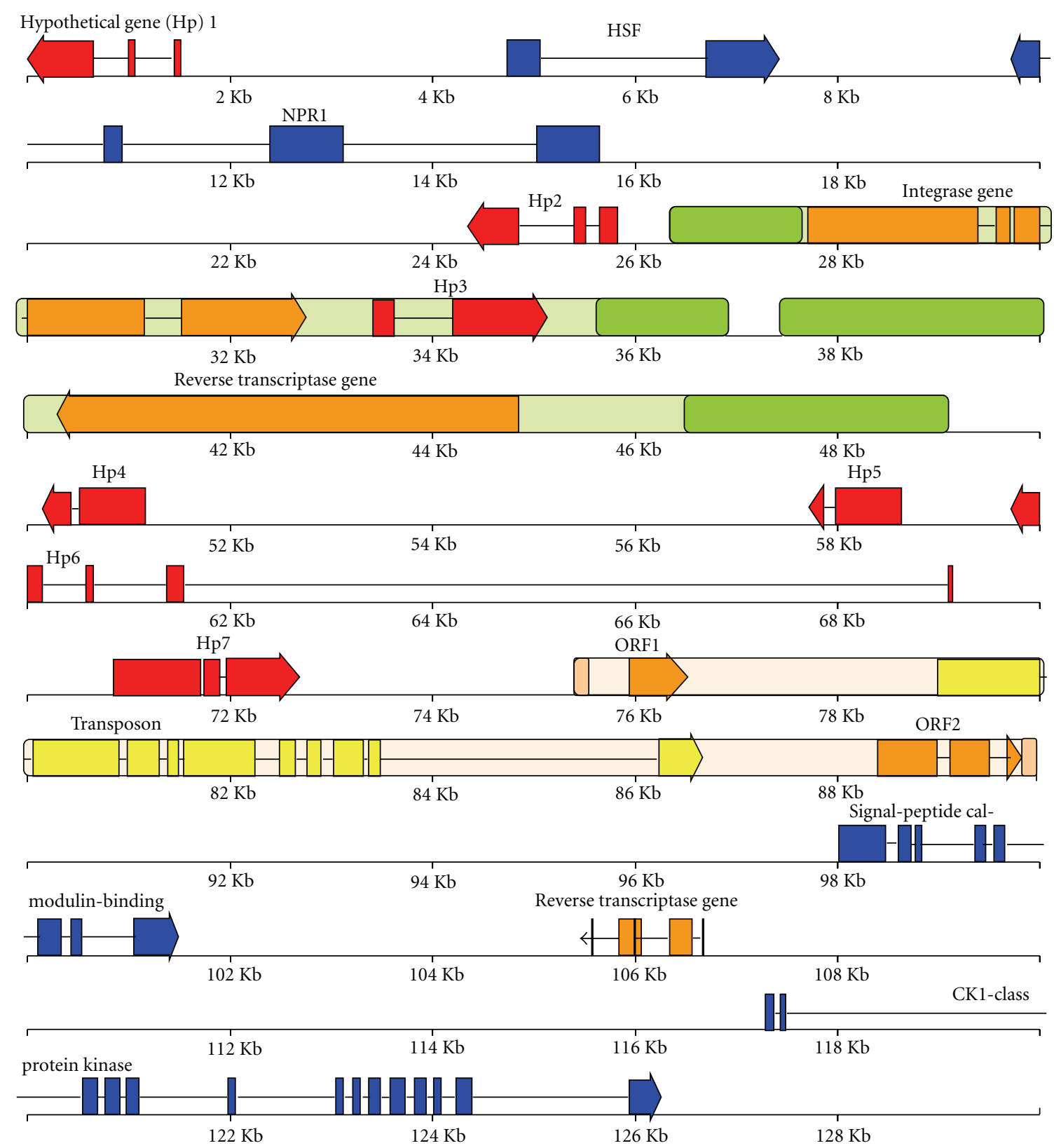

FIGURE 1: BAC physical map showing two LTR retrotransposons SCHULTE and SCHMIDT both highlighted in green and with darker green LTRs with rounded corners. On the other hand, the previously reported CACTA transposon Coel, highlighted in light beige, has short tan LTRs, a yellow DNA transposase gene and orange ORF1 and ORF2. Exons are rectangles, and direction of arrows indicates direction of transcription. Exons of core plant genes are blue, and exons of hypothetical protein genes are red. Solid lines between exons depict introns. Scale is in $2 \mathrm{~Kb}$ increments. BAC is about 130 kilobase pairs. SCHULTE has an integrase gene in orange and a hypothetical gene in red. SCHMIDT has a single ORF retroelement reverse transcriptase polyprotein gene in orange.

was clearly evidenced by alignments, $E=0.0$, with ESTs BI698297 and BI698341. Four other ESTs showing some alignment $\left(E=\mathrm{e}^{-151}\right.$ to $\left.E=9 \mathrm{e}^{-36}\right)$ also suggest other likely active SCHULTE-like elements.

Another LTR retrotransposon discovered using LTR_STRUC and LTR FINDER, SCHMIDT was so named to honor a pioneering researcher of repeat DNA elements in Beta. SCHMIDT, $11565 \mathrm{bp}$ retroelement, encodes a complete Ty3-gypsy-class polyprotein in a single ORF without introns. The SCHMIDT reverse transcriptase gene has all of the domains expected of an intact retroelement polyprotein, and the domain order is indicative of Ty3gypsy-class. SCHMIDT has 2561 bp LTR sequences with $100 \%$ identity, consistent with this transposable element still being active.

EMBOSS analysis of the $130 \mathrm{~Kb} N P R 1$-carrying sugarbeet $\mathrm{BAC}$ revealed the presence of at least 24 inverted repeat (IR) sequences but, for the purposes of this report, let us 

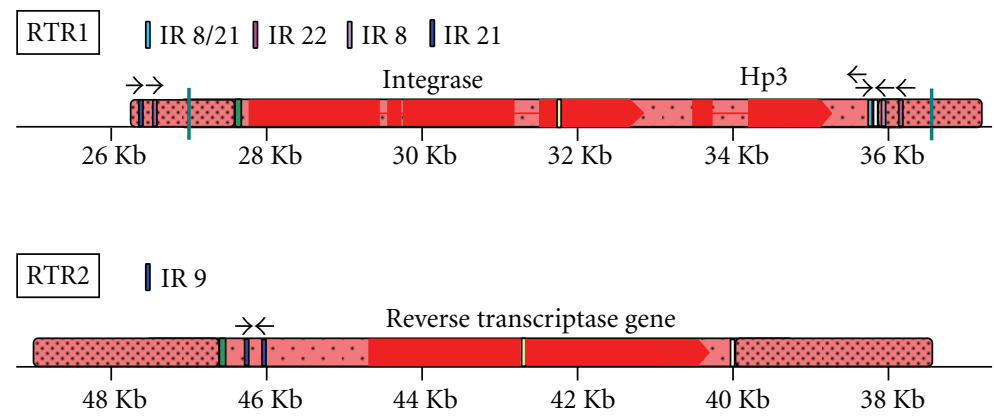

$\begin{array}{ll}0 \text { Active site } & \\ 0 \text { PBS } & \text { LTRs } \\ 0 \text { PTRs } \\ \text { I Colypurine tract } & \\ \text { I Exons }\end{array}$

FIGURE 2: A detailed schematic of Beta vulgaris retrotransposons SCHULTE and SCHMIDT in red. Various numbered inverted repeat (IR) sequences in either light or dark blue or violet have arrows indicating relative direction. Green lines show the location of the DNA sequence motif CACTATAA. Heavily dotted ovular regions depict the size and position of the LTRs. The lightly dotted region shows the size of the whole retroelement. A green box shows the location of the polymerase binding site. Boxes show the position and size of exons. Lines between exons indicate introns. A centrally located small yellow box depicts the active site of the retroelement. An orange box shows the location of the polypurine tract. Scale is in $\mathrm{Kb}$ and is located underneath the illustrations in increments of $2 \mathrm{~Kb}$ per tick. Names of putative genes are located above the illustrations.

describe only those inverted sequences associated with LTR retrotransposons SCHULTE and SCHMIDT. The following two pairs of $I R 8 / 21$ inverted repeat sequences are associated with SCHULTE (Figure 2). The IR $8 / 21$ inverted repeat sequences share $94 \%$ identity $(18 / 19)$.

$$
\begin{gathered}
35690 \text { cttagtttgtacctttgtt } 35708 \\
|||||||||||||||||| \mid \\
35781 \text { gaatcaaacaaggaacaa } 35763 \\
26302 \text { aacaaaggaacaaactaag } 26320 \\
\text { || |||||| || || || || || } \\
35708 \text { ttgtttccatgtttgattc } 35690
\end{gathered}
$$

The following pair of IR 22 inverted sequences, associated with SCHULTE (Figure 2), are $9.5 \mathrm{~Kb}$ apart and share $80 \%$ identity (shown below), but this pair of IR 22 are also direct repeats with $96 \%$ identity.

$$
\begin{aligned}
& 26751->26786 \\
& \text { aaaaagaaatctgttttggaaagattttatttt } \\
& |||||||||||||||||||||||||||||| \mid \\
& \text { tttttattttagaaaagattttgtctaaaagaaaa } \\
& 36247<-36212
\end{aligned}
$$

SCHMIDT has a pair of inverted repeat IR 9 sequences upstream of the single reverse transcriptase polyprotein gene and just downstream of polymerase binding site (Figure 2). These IR 9 inverted repeat sequences share 100\% identity $(23 / 23)$ and contain only nucleotides A and T.

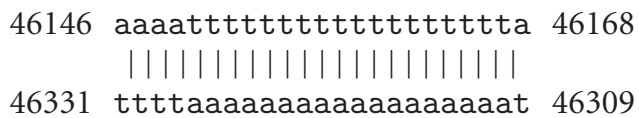

Over about one-tenth of their length, each LTR sequence of SCHMIDT shares $97 \%$ nucleic acid sequence identity with $p D R V$, a family of Beta vulgaris short repeated DNA sequences known as rich in DraI (TTTAAA) restriction enzyme recognition sites [5]. For example, eight DraI sites are carried by $p D R V 1$, a $434 \mathrm{bp}$ repeated sequence [5]. Each LTR of SCHMIDT has 10 DraI sites, and the fulllength SCHMIDT retrotransposon has twenty-four DraI (TTTAAA) sites. Perhaps $p D V R$ conserved sequences were originally a preferred recognition site for insertion; they seem to have evolved into an integral part of the LTRs of SCHMIDT-like retrotransposons. In any case, the observed high degree of nucleotide sequence identity that the LTRs of SCHMIDT have with pDRVs, highly reiterated sequences rich in nucleotides $\mathrm{A}$ and $\mathrm{T}$ [5], is very interesting since $p D R V$ repeat sequences, originally visualized by FISH, are dispersed over all 18 sugarbeet chromosomes [5].

Figure 5 shows results of alignment by MegAlign of conserved rve and Rvt1 domains of the protein products from SCHMIDT-like plant retrotransposons identified by BLASTp from various plant species, and Figure 6 shows a neighbor joining analysis tree by MEGA 4 showing structural relatedness. A highly conserved FIDDILI active site in the $R v t 1$ domain is noted in particular (Figure 5). The predicted SCHMIDT reverse transcriptase polyprotein gene product shows high structural similarity with a conserved region of proteins encoded by similar LTR retrotransposons from Cicer arietinum, Medicago truncatula, Oryza sativa japonica, Sorghum bicolor, and Zea mays AAL59229. Somewhat similar LTR retrotransposons from Glycine max, Primula vulgaris, Vitis vinifera, Solanum demissum, Hordeum vulgare, and Zea mays AAM94350 produce structurally divergent products (Figure 6). Against the TIGR plant repeat database, SCHMIDT produced good, $E \leq \mathrm{e}^{-50}$, BLAST nucleotide 


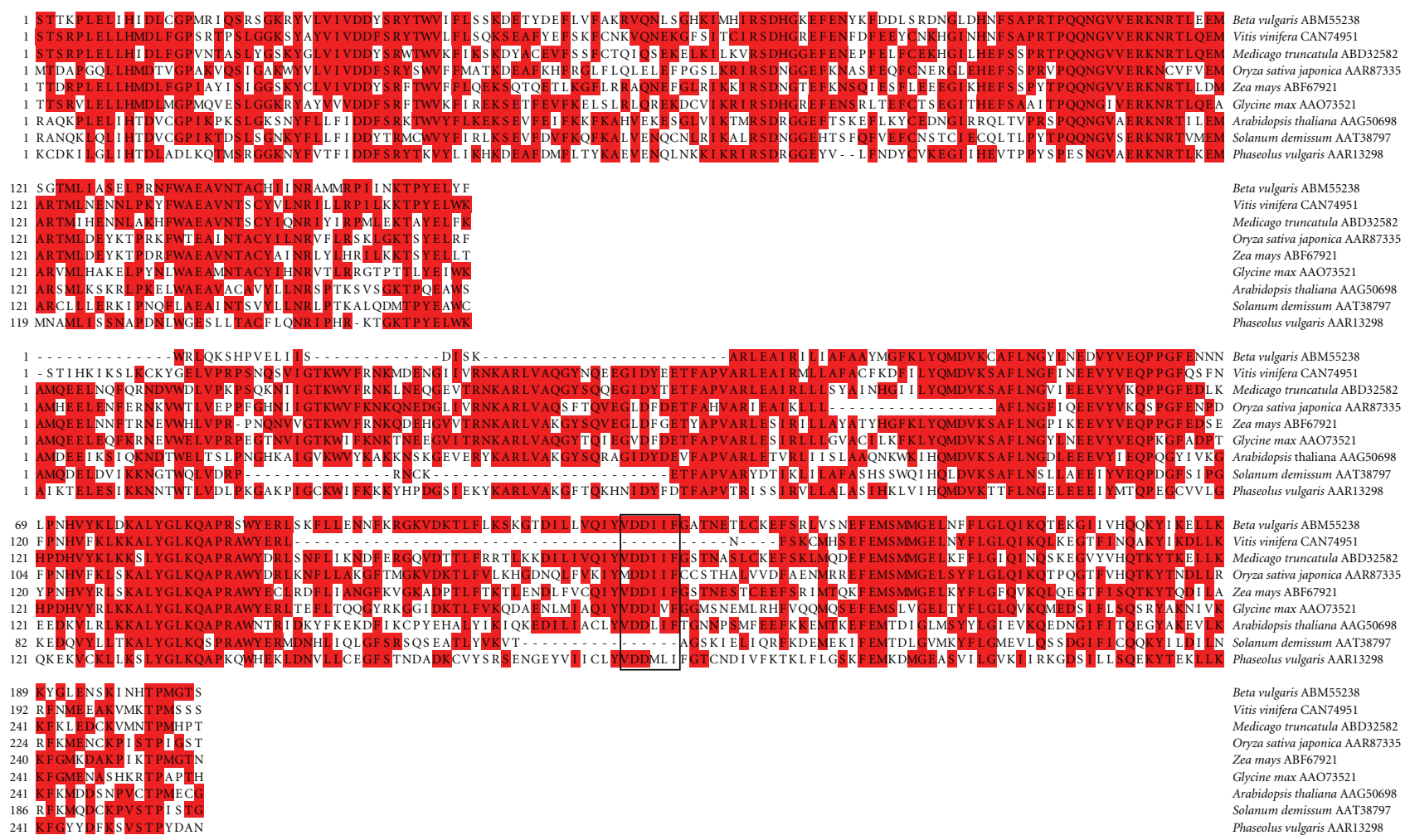

Figure 3: Amino acid residue alignment of SCHULTE's integrase (top) and reverse transcriptase (bottom) domains. The active site of the RT domain from Beta vulgaris is boxed in along with corresponding sections of other reverse transcriptase proteins from different plants. Amino acids matching the consensus sequence are shaded. Numbers indicate cumulative amino acid positions; Arabidopsis thaliana (AAG50698), Beta vulgaris (ABM55238), Glycine max (AAO73521), Medicago truncatula (ABD32582), Oryza sativa japonica (AAR87335), Phaseolus vulgaris (AAR13298), Solanum demissum (AAT38797), Vitis vinifera (CAN74951), Zea mays (ABF67921).

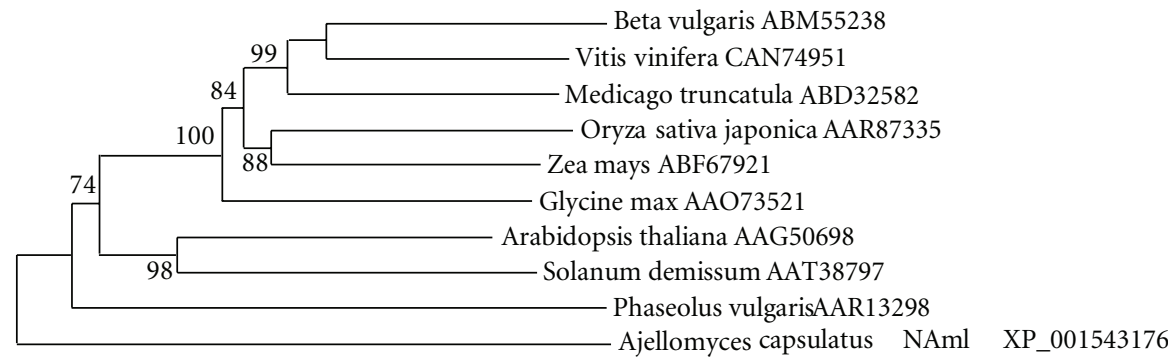

FIGURE 4: Similarity tree constructed by neighbor joining method of the reverse transcriptase domain of SCHULTE from Beta vulgaris along with corresponding domains of other reverse transcriptase proteins from different plants. The numbers on the branches are bootstrap (confidence) values. Genbank accession numbers of amino acid sequences are given following plant names.

sequence identity which matches primarily with Prem1and Xilon1-like gypsy-like RTRs in Zea, Oryza, Sorghum, and Triticum. This finding suggests divergent evolution, where a SCHMIDT-like ancestor originated in monocots, then, upon lateral transfer to sugarbeet, natural selection for structural similarity or convergence in a new genetic background resulted in a high degree of amino acid similarity of the protein product with other gypsy-like retrotransposons in eudicots. The predictions of convergent evolution are structurally similar proteins encoded by phylogenetically distinct retrotransposons. Whether similar sequences arose through convergent or divergent evolution, it is interesting to simply note that SCHMIDT has a significant degree of nucleotide sequence identity primarily with certain gypsy-like retrotransposons found in monocots.

Expression of retroelements similar to SCHMIDT in sugarbeets is suggested by the finding that SCHMIDT gave BLAST alignments with the following ESTs: BI643170, BI643158, BI698360, and BI643246 $\left(E=10^{-161}, 3 E=10^{-79}\right.$, $E=10^{-68}$, and $5 E=10^{-59}$, resp.). These four BLAST hits represent only about $0.02 \%$ of the ESTs in the collection. 


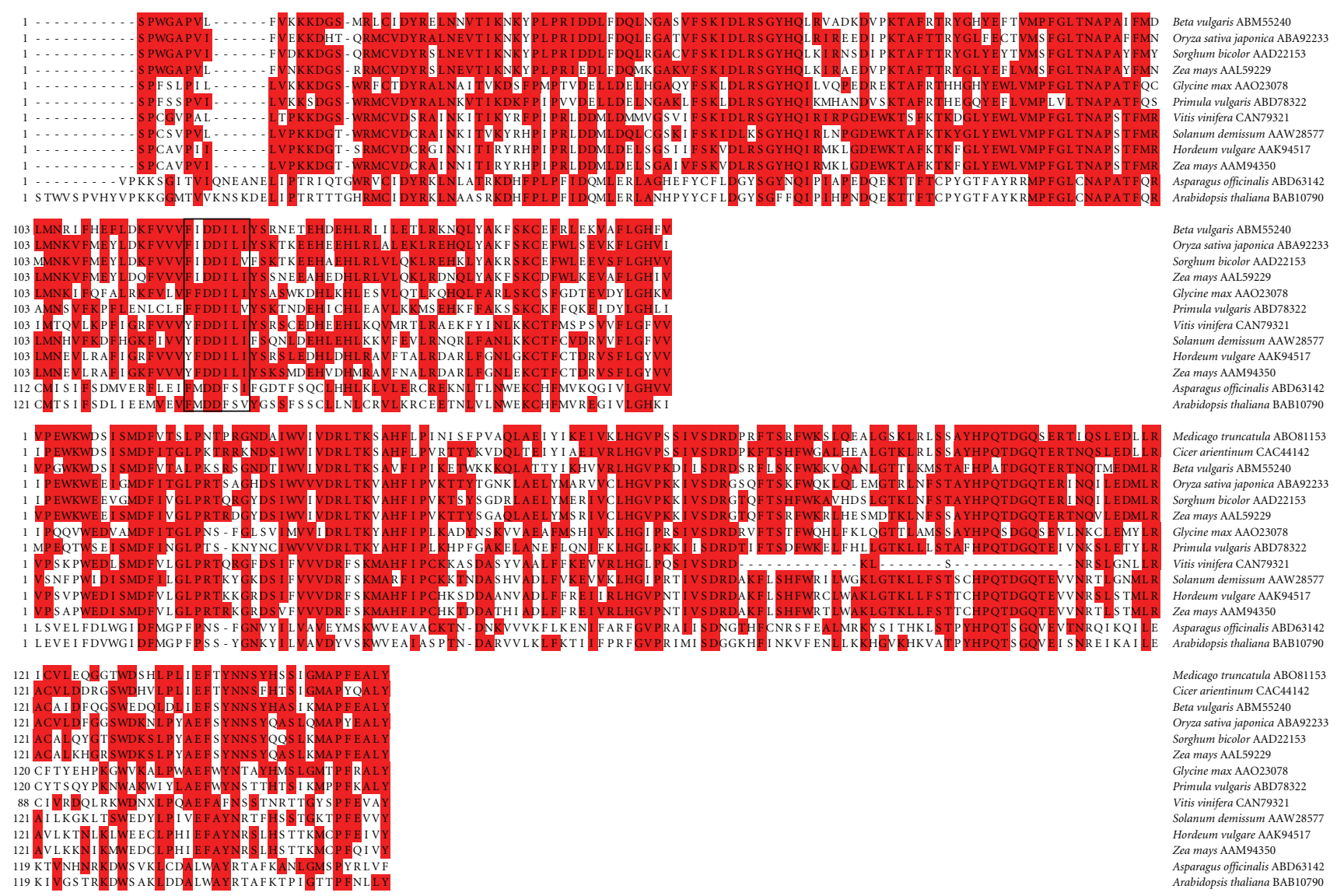

Figure 5: Amino acid residue alignment of the reverse transcriptase (top) and integrase (bottom) domains of SCHMIDT from Beta vulgaris, with its active site boxed in, along with corresponding sections of other reverse transcriptase proteins from different plants. Amino acids matching the consensus sequence are shaded. Numbers indicate cumulative amino acid positions; Arabidopsis thaliana (BAB10790), Asparagus officinalis (ABD63142), Beta vulgaris (ABM55240), Cicer arietinum (CAC44142), Glycine max (AAO23078), Hordeum vulgare (AAK94517), Medicago truncatula (ABO81153), Oryza sativa japonica (ABA92233), Primula vulgaris (ABD78322), Solanum demissum (AAW28577), Sorghum bicolor (AAD22153), Vitis vinifera (CAN79321), Zea mays (AAM94350), Zea mays (AAL59229).

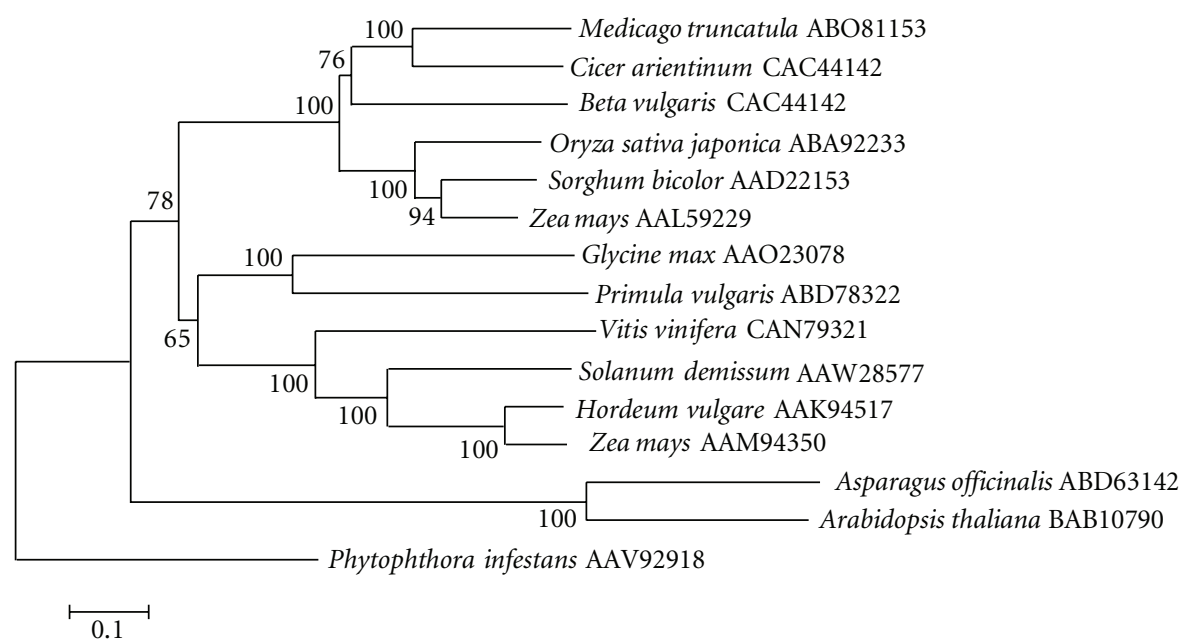

FIGURE 6: Similarity tree constructed by neighbor joining method of the reverse transcriptase domain of SCHMIDT from Beta vulgaris along with corresponding domains of other reverse transcriptase proteins from different plants. The numbers on the branches are bootstrap (confidence) values. Genbank accession numbers of amino acid sequences are given following plant names. 
TABLE 1: Several LTR retrotransposons discovered within a TE nest, in addition to Coe1 [7], by LTR_STRUC analysis of a sugarbeet genomic BAC carrying NPR1.

\begin{tabular}{|c|c|c|c|c|}
\hline Feature & SCHULTE & SCHMIDT & Older LTR-RTR & Coe1 [7] \\
\hline Active site & YVDDIIL & FIDDILI & SCDDVLL & YVDDIIL \\
\hline Length of RTR & $10833 \mathrm{bp}$ & $11565 \mathrm{bp}$ & $\begin{array}{l}5395 \text { bp (before two } \\
\text { TE insertions) }\end{array}$ & $14531 \mathrm{bp}$ \\
\hline Length of LTR & $1372 \mathrm{bp}$ & $2561 \mathrm{bp}$ & $780 \mathrm{bp}$ & $169 \mathrm{bp}$ \\
\hline 5' LTR-3' LTR Identity (\%) & $99.3 \%$ & $100.0 \%$ & $99.0 \%$ & $96.4 \%$ \\
\hline $\begin{array}{l}\text { Number of open reading } \\
\text { frames (ORFs) }\end{array}$ & 2 & 1 & 2 & 3 \\
\hline $\begin{array}{l}5^{\prime} \text { beginning and } 3^{\prime} \text { end of } \\
\text { flanking region (duplication) }\end{array}$ & ATTTT & CGCTC & GCTTG & CTACT \\
\hline Class \& domains present & $\begin{array}{l}\text { Copia-like } \\
\text { Integrase and } \\
\text { RTase domains } \\
\end{array}$ & $\begin{array}{l}\text { All domains expected of } \\
\text { a complete Gypsy-like } \\
\text { retrotransposon }\end{array}$ & Putative RT domains & $\begin{array}{l}\text { Coe1 [7], class II within a class } \\
\text { I, DNA transposase/Copia-like } \\
\text { RTase pseudogene }\end{array}$ \\
\hline
\end{tabular}

An older LTR retrotransposon, which had been interrupted by subsequent insertions of SCHULTE and SCHMIDT, became evident (Table 1) when LTR_STRUC analysis was performed on a sequence having an in silico deletion of the LTR retrotransposons SCHULTE and SCHMIDT. Although very degraded and unclassifiable, the older LTR retrotransposon was deduced to be $5395 \mathrm{bp}$ with 780 bp LTRs sharing 99\% identity.

In conclusion, the relatively small repetitive DNA sequences previously described as " $p D R V$ s" can now be seen as a part of the LTRs of SCHMIDT-like retrotransposons.

Planned research will address possible effects of retrotransposons on the expression of core plant genes including the NPR1 disease resistance-priming gene immediately downstream of the LTR retrotransposon nest.

\section{Conclusions}

An LTR retrotransposon nest consisting of an older retroelement into which both a gypsy-like SCHMIDT and a copialike SCHULTE inserted was identified, and properties of the retrotransposons were described. Since LTR retrotransposons are driving forces in plant genome evolution (see reviews $[1,2]$ ), they may have tremendous potential usefulness in genetic manipulation and genome modification to enhance agricultural profitability and sustainability.

\section{References}

[1] J. L. Bennetzen, "Transposable elements, gene creation and genome rearrangement in flowering plants," Current Opinion in Genetics and Development, vol. 15, no. 6, pp. 621-627, 2005.

[2] J. M. Casacuberta and N. Santiago, "Plant LTR-retrotransposons and MITEs: control of transposition and impact on the evolution of plant genes and genomes," Gene, vol. 311, no. 1-2, pp. 1-11, 2003.

[3] T. Schmidt, S. Kubis, and J. S. Heslop-Harrison, "Analysis and chromosomal localization of retrotransposons in sugar beet (Beta vulgaris L.): LINEs and Ty1-copia-like elements as major components of the genome," Chromosome Research, vol. 3, no. 6, pp. 335-345, 1995.
[4] D. Dechyeva, F. Gindullis, and T. Schmidt, "Divergence of satellite DNA and interspersion of dispersed repeats in the genome of the wild beet Beta procumbens," Chromosome Research, vol. 11, no. 1, pp. 3-21, 2003.

[5] T. Schmidt, S. Kubis, A. Katsiotis, C. Jung, and J. S. HeslopHarrison, "Molecular and chromosomal organization of two repetitive DNA sequences with intercalary locations in sugar beet and other Beta species," Theoretical and Applied Genetics, vol. 97, no. 5-6, pp. 696-704, 1998.

[6] G. Jacobs, D. Dechyeva, G. Menzel, C. Dombrowski, and T. Schmidt, "Molecular characterization of Vulmar1, a complete mariner transposon of sugar beet and diversity of marinerand En/Spm-like sequences in the genus Beta," Genome, vol. 47, no. 6, pp. 1192-1201, 2004.

[7] D. Kuykendall, J. Shao, and K. Trimmer, "Coe1 in Beta vulgaris L. has a Tnp2-domain DNA transposase gene within putative LTRs and other retroelement-like features," International Journal of Plant Genomics, vol. 2008, Article ID 360874, 7 pages, 2008.

[8] D. Kuykendall, J. Shao, and T. Murphy, "Conserved microsynteny of NPR1 with genes encoding a signal calmodulinbinding protein and a CK1-class protein kinase in Beta vulgaris and two other eudicots," International Journal of Plant Genomics, vol. 2008, Article ID 391259, 8 pages, 2008.

[9] D. Kuykendall, T. S. Murphy, J. Shao, and J. M. McGrath, "Nucleotide sequence analyses of a sugarbeet genomic NPR1class disease resistance gene," Journal of Sugar Beet Research, vol. 44, pp. 35-49, 2007.

[10] J. M. McGrath, R. S. Shaw, B. G. de los Reyes, and J. J. Weiland, "Con-struction of a sugarbeet BAC library from a hybrid that combines diverse traits," Plant Molecular Biology Reporter, vol. 22, no. 1, pp. 23-28, 2004.

[11] E. M. McCarthy and J. F. McDonald, "LTR_STRUC: a novel search and identification program for LTR retrotransposons," Bioinformatics, vol. 19, no. 3, pp. 362-367, 2003.

[12] Z. Xu and H. Wang, "LTR_FINDER: an efficient tool for the prediction of full-length LTR retrotransposons," Nucleic Acids Research, vol. 35, web server issue, pp. 265-268, 2007.

[13] P. Rice, L. Longden, and A. Bleasby, "EMBOSS: the european molecular biology open software suite," Trends in Genetics, vol. 16, no. 6, pp. 276-277, 2000.

[14] R. Herwig, B. Schulz, B. Weisshaar, et al., "Construction of a 'unigene' cDNA clone set by oligonucleotide fingerprinting 
allows access to 25000 potential sugar beet genes," The Plant Journal, vol. 32, no. 5, pp. 845-857, 2002.

[15] D. Schulte, D. Cai, M. Kleine, L. Fan, S. Wang, and C. Jung, "A complete physical map of a wild beet (Beta procumbens) translocation in sugar beet," Molecular Genetics and Genomics, vol. 275, no. 5, pp. 504-511, 2006.

[16] P. A. Reeves, Y. He, R. J. Schmitz, R. M. Amasino, L. W. Panella, and C. M. Richards, "Evolutionary conservation of the FLOWERING LOCUS C-mediated vernalization response: evidence from the sugar beet (Beta vulgaris)," Genetics, vol. 176, no. 1, pp. 295-307, 2007. 

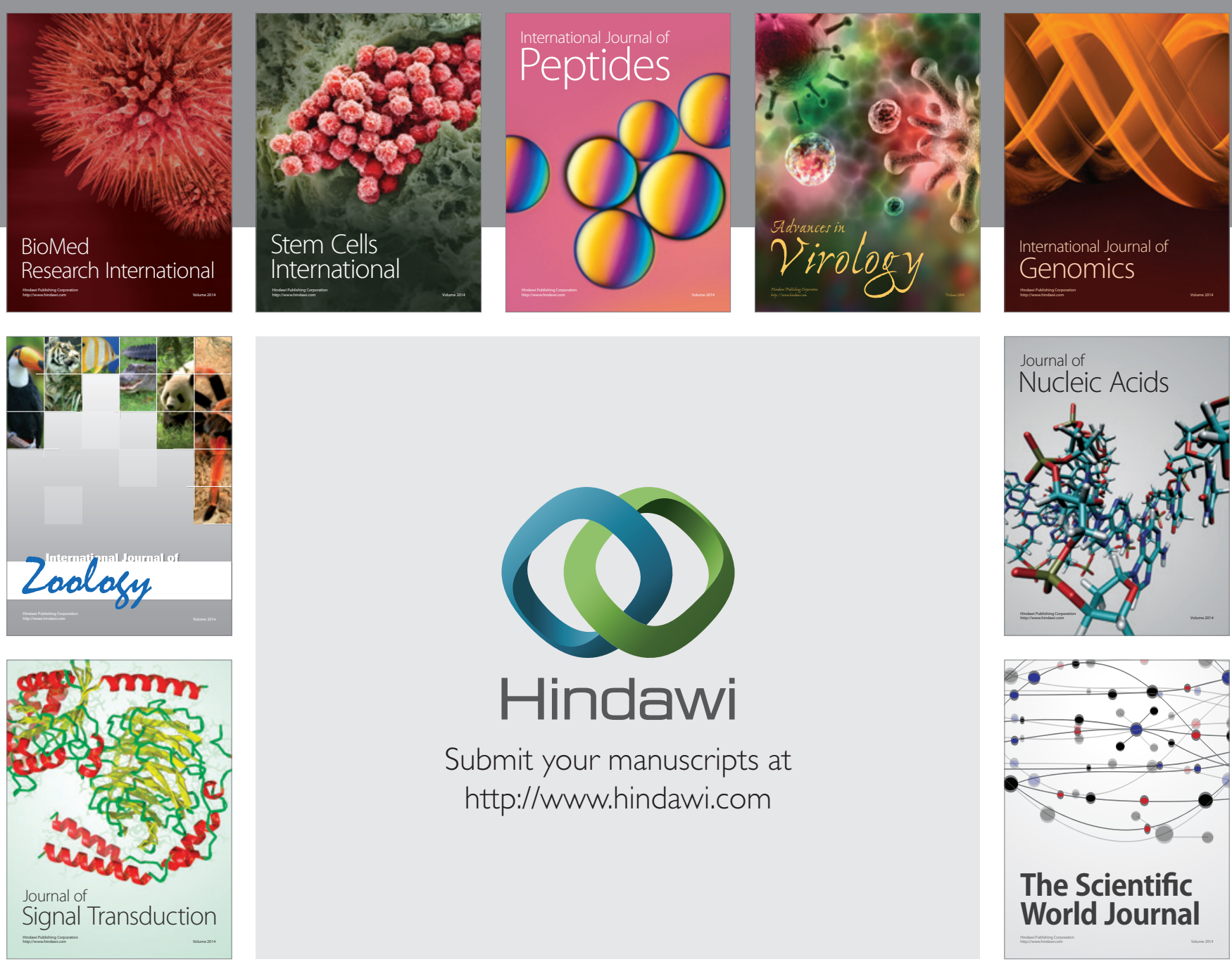

Submit your manuscripts at

http://www.hindawi.com
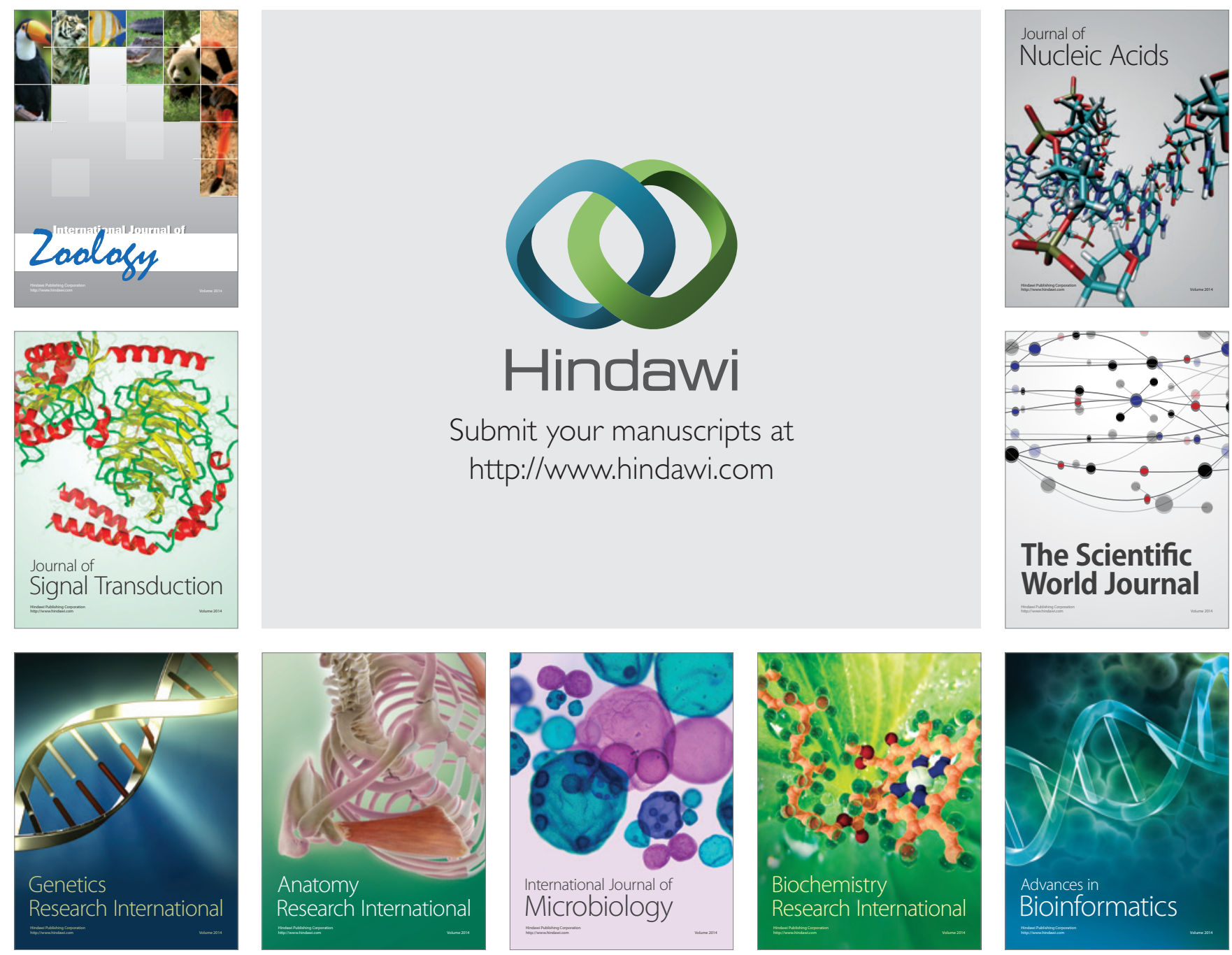

The Scientific World Journal
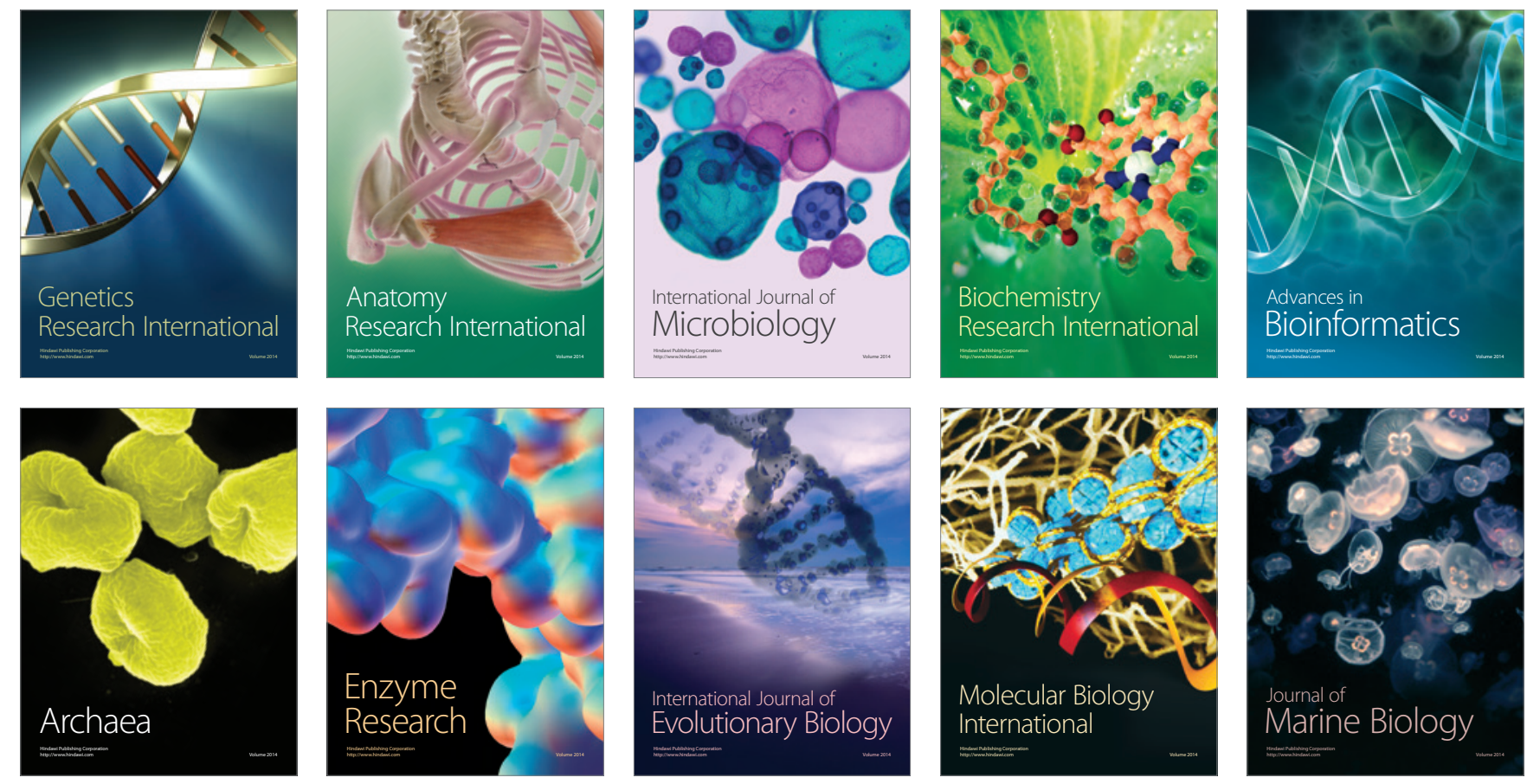